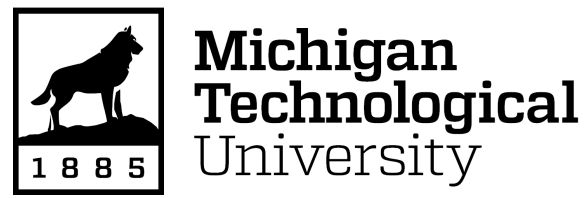

Michigan Technological University Digital Commons @ Michigan Tech

Dissertations, Master's Theses and Master's Reports

2017

\title{
CONTROL OF A POWERED ANKLE-FOOT PROSTHESIS: FROM PERCEPTION TO IMPEDANCE MODULATION
}

Guilherme Aramizo Ribeiro

Michigan Technological University, garamizo@mtu.edu

Copyright 2017 Guilherme Aramizo Ribeiro

\section{Recommended Citation}

Aramizo Ribeiro, Guilherme, "CONTROL OF A POWERED ANKLE-FOOT PROSTHESIS: FROM PERCEPTION TO IMPEDANCE MODULATION", Open Access Master's Thesis, Michigan Technological University, 2017. https://doi.org/10.37099/mtu.dc.etdr/405

Follow this and additional works at: https://digitalcommons.mtu.edu/etdr

Part of the Biomechanical Engineering Commons, Controls and Control Theory Commons, and the Robotics Commons 
CONTROL OF A POWERED ANKLE-FOOT PROSTHESIS: FROM PERCEPTION TO IMPEDANCE MODULATION

\author{
By \\ Guilherme A. Ribeiro

\begin{abstract}
A REPORT
Submitted in partial fulfillment of the requirements for the degree of MASTER OF SCIENCE

In Mechanical Engineering

MICHIGAN TECHNOLOGICAL UNIVERSITY

2017
\end{abstract}

(C) 2017 Guilherme A. Ribeiro 
This report has been approved in partial fulfillment of the requirements for the Degree of MASTER OF SCIENCE in Mechanical Engineering.

Department of Mechanical Engineering-Engineering Mechanics

Report Advisor: $\quad$ Mo Rastgaar

Committee Member: $\quad$ Ossama O. Abdelkhalik

Committee Member: Timothy C. Havens

Committee Member: Nina Mahmoudian

Department Chair: William W. Predebon 


\section{Table of Contents}

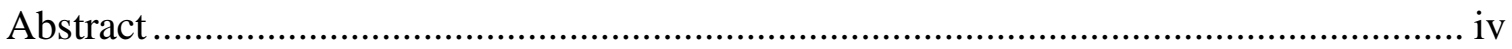

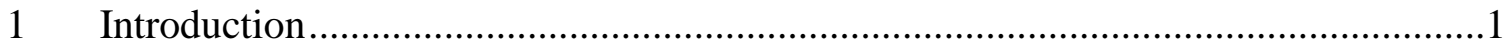

2 Inertia Estimation using the Instrumented Vibrating Platform..................................2

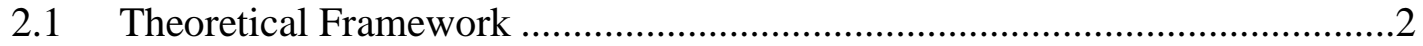

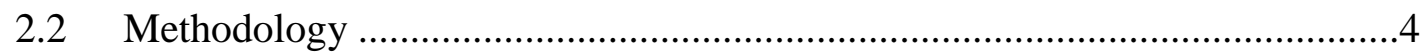

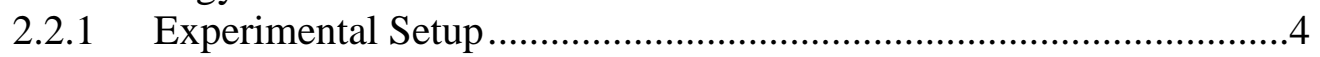

2.2.2 Dynamic Simulation .....................................................................

2.2.3 Parameter Estimation Algorithm ..................................................6

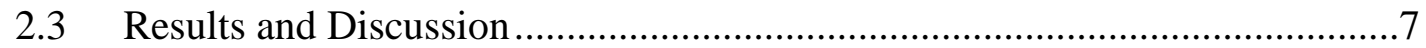

3 Wearable Vision System for Ground Mapping …………......................................

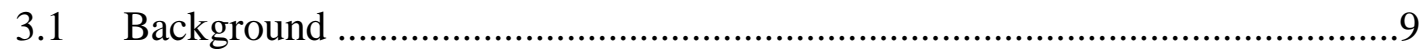

3.1.1 Depth Sensing Devices ..................................................................

3.1.2 Simultaneous Localization and Mapping .........................................10

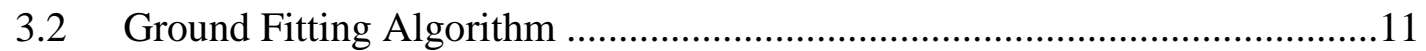

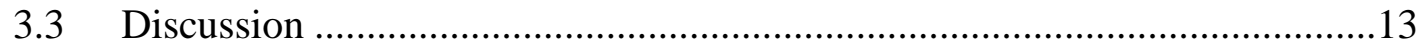

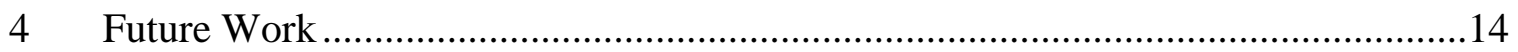

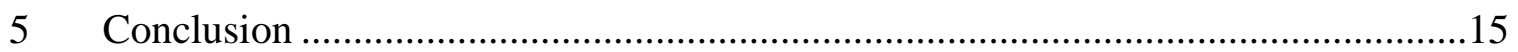

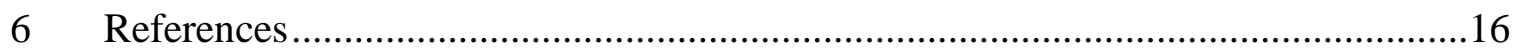




\section{Abstract}

Active ankle prostheses controllers are gaining smart features to improve the safety and comfort of users. The perception of user intention to modulate the ankle dynamics is a wellknown example of such feature. But not much work focused on the perception of the environment, nor how the environment should be included in the mechanical design and control of the prostheses. The proposed work aims to integrate environment perception to prostheses controllers and to define the desired ankle dynamics of the human walk on different environmental settings. As a preliminary work on environment perception, a vision system was developed that can estimate the ground slope and height. The desired prosthesis dynamics was defined as the mechanical impedance of a healthy ankle, which required the system identification of the human ankle. Simulations showed the inertia parameters of a mockup foot can be estimated. Further experiments will show the accuracy of environment perception and of the impedance estimation. 


\section{Introduction}

Intelligent methods to control active prostheses devices are a key factor to achieve safe and transparent operation for amputee's users. To do so, many prostheses controllers rely on user intention and the environment perception to control the prosthesis actuators [1]. The perception and execution features of prosthesis controllers are commonly divided into layers due to their different roles.

On the perception layer, the user intention perception is often implemented using standard prosthesis sensors (inertial measurements of the prostheses and interaction forces [2-4]) to estimate an ambulation state (walking, standing, sitting, stair climb, etc). But not as much attention has been given to environment perception.

The importance of environment perception is highlighted considering how walking kinematics and kinetics change depending on the ground slope [5] and flatness [6]. This problem of modulating a prosthesis for different ground slopes was addressed by Sup [7], who developed a ground slope estimator and modulated their impedance controller accordingly. Their slope estimator used inertial readings during the stance phase, thus, the slope estimated was always delayed by 1 step.

The delay in the environment perception via proprioceptive sensors is well-known. Krausz, Lenzi [8] used a Microsoft Kinect sensor to identify the proximity to and geometry of a staircase for use in a lower limb prosthesis. Liu, Wang [9] used inertial and laser range measurements to classify walking modes as up/down ramp/stair, and leveled walk. Overall, recent developments in ranging sensors are enabling prostheses to perceive the environment in a timelier manner.

On the execution layer, control engineers often opt for a controller with ankle compliance during the stance phase, such as emulating a stiffness [10] or an impedance [7] of a healthy walk. Additionally, the ankle has been reported to change its impedance across the cycle of the straight walk [11-13]. But prosthesis controllers cannot address these problems because there is no reported work quantifying the impedance as a function of ground slopes or gait maneuver.

Therefore, there are 2 problem fronts: the perception layer must identify more accurately and timely the environment, while the execution layer must know how to operate the robotic ankle given the perception output. These issues are addressed in this report with a preliminary work on 1) estimating the ankle impedance in different walking conditions, in Chapter 2; and 2) developing a vision system to improve environment perception of prostheses, in Chapter 3. 


\section{Inertia Estimation using the Instrumented Vibrating Platform}

An instrumented vibrating platform was developed in a previous study to estimate the mechanical impedance of the human ankle [14]. It is composed of a motion capture system to record the ankle's kinematics, and a force plate (FP) mounted on top of a vibrating platform to both record the ankle's kinetics and apply perturbations to the system. The ground perturbations, occurring in the sagittal and frontal planes of the ankle, frees the subject from carrying a heavy test equipment, which allows the subject to perform a more natural walk.

The next step of this work is to estimate the impedance of a human during walk, which was reported to have time-varying characteristics through the gait cycle [11-13]. Another step is to separate the intrinsic impedance from the nonlinear reflexive component. The intrinsic component can be estimated by observing the ankle dynamics only during the initial moments of the perturbation, before the reflexive component starts acting [15].

Therefore, to estimate a time-varying impedance that works with short-time perturbations, a new impedance estimation method was developed. This method fits the system parameters, such as inertia, stiffness, and damping to the translational and rotational equations of motion of the system. An initial validation step is to estimate the inertia of the vibrating platform and a mockup foot, without the effect of the ankle stiffness and damping, and the person's active balance control [16].

In this work, the equations of motion of the vibrating platform were derived, validated against a numerical simulation, and used to estimate the inertia parameters of the vibrating platform and of a mockup human's foot on experimental and simulation data. Most of the inertia parameters were estimated accurately with simulation data, but results from experimental analysis indicate the need for modifying the testing protocol.

\subsection{Theoretical Framework}

The proposed procedure aims to estimate the 10 inertia parameters of the force plate (FP) first in one experiment, then use the results to estimate the foot mockup inertia in a second experiment. The experimental protocol is the same in both cases: the platform is perturbed by a pseudo-random input, the motion capture system records the kinematics data, the force plate records kinetics data, and the inertial parameters are fit according to the equations of motion.

Similar methods to estimate all the 10 inertia parameter of a rigid body (mass, center of gravity vector, 3 moments of inertia, and 3 products of inertia) have been presented in [17]. The proposed method can be classified as direct parameter estimation via base excitation, which has been used by other investigators $[18,19]$, who also reported good accuracy. Like 
these methods, the analytical description of the system is developed and then the numerical method is presented.

For the mathematical modeling of the ankle impedance system, 3 coordinates systems were defined: the inertial frame $\mathcal{J}$, the plate frame $\mathcal{P}$, and the foot frame $\mathcal{F}$ (Figure 1 ). The $\mathrm{X}$ axis and $\mathrm{Z}$ axis are normal to frontal and sagittal frames, respectively.

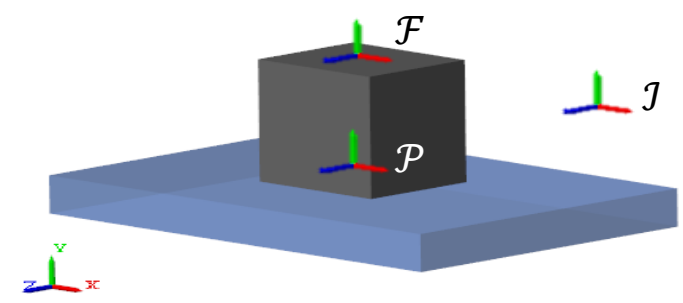

Figure 1. Coordinate frame definition of the ankle impedance

The force plate (FP) motion is constrained by a universal joint, allowing a rotation on the inertial $\mathrm{Z}$ axis and on the plate $\mathrm{X}$ axis. $R_{\mathcal{P} \mathcal{J}} \in \mathbb{R}^{3} \times \mathbb{R}^{3}$ and $p_{\mathcal{P} \mathcal{J}} \in \mathbb{R}^{3}$ is the rotation matrix and translation vector from the $\mathcal{J}$ to $\mathcal{P}$ frame, respectively.

The frame $\mathcal{F}$ attached to the mockup foot allows the estimation of the foot properties, independent of its position and orientation in respect to the $\mathcal{P}$ frame. $R_{\mathcal{F} \mathcal{P}} \in \mathbb{R}^{3} \times \mathbb{R}^{3}$ and $p_{\mathcal{F P}} \in \mathbb{R}^{3}$ is the rotation matrix and translation vector from the $\mathcal{P}$ to $\mathcal{F}$ frame, respectively.

The position of the FP's center of gravity (CG) in the $\mathcal{P}$ frame, ${ }_{\mathcal{P}} p_{p}=\left(x_{p 0}, y_{p 0}, z_{p 0}\right)^{T} \in$ $\mathbb{R}^{3}$ is defined in respect to the origin of the $\mathcal{P}$ frame. From that and disregarding the foot, the Newton's Second Law applied on the FP yields

$$
m_{p} \ddot{p}_{p}=\sum F=m_{p} g+\hat{F}_{0}+F_{s}
$$

where

1. $m_{p} \in \mathbb{R}$ is the FP mass.

2. $g \in \mathbb{R}^{3}$ is the gravity vector pointing downwards.

3. $\widehat{F}_{0} \in \mathbb{R}^{3}$ is the scale tare weight subtracted from the FP measurement.

4. $F_{s} \in \mathbb{R}^{3}$ is the FP force measurement.

The tare subtracts the load cell bias and the static weight of the FP. Therefore, ${ }_{\mathcal{P}} \widehat{F}_{0}=$ $-R_{P I} m_{p} g$ can be modeled as a constant force on the $\mathcal{P}$ frame, which expands the translational equation of motion to

$$
m_{p} \ddot{p}_{p}-m_{p} g+R_{\mathcal{P J}} m_{p} g=F_{s}
$$


Similarly, the Euler's Equations calculated at the surface of the FP, in the $\mathcal{P}$ frame is

$$
J_{p}{ }^{\mathcal{P}} \dot{\omega}_{p}+\omega_{p} \times\left(J_{p} \omega_{p}\right)=\sum T=T_{s}+d_{p} \times\left(m_{p} \ddot{p}_{p}+m_{p} g\right)
$$

where

1. $J_{p} \in \mathbb{R}^{3} \times \mathbb{R}^{3}$ is the FP inertia tensor measured at the CG.

2. $\omega_{p} \in \mathbb{R}^{3}$ is the angular velocity of the $\mathcal{P}$ frame.

3. $T_{s} \in \mathbb{R}^{3}$ is the FP torque measurement.

4. $d_{p} \in \mathbb{R}^{3}$ is the distance from the FP origin to the FP CG in the $\mathcal{P}$ frame.

5. The left superscript $\mathcal{P}$ denote a derivative on the non-inertial frame $\mathcal{P}$.

Therefore, the rotational equation of motion is

$$
J_{p}{ }^{\mathcal{P}} \dot{\omega}_{p}+\omega_{p} \times\left(J_{p} \omega_{p}\right)-d_{p} \times\left(m_{p} \ddot{p}_{p}+m_{p} g\right)=T_{s}
$$

Because the kinematic variables $\left(\ddot{p}_{p}, \omega_{p}, \dot{\omega}_{p}\right)$ are constrained by the universal joint, they are dependent of the FP angles $\theta, \varphi$, their first and second derivatives. But for simplicity, equations (1.a-b), and further equations are not expanded.

Similarly, if the foot is added on top of the FP, the inertial and weight components of the Equations (1.a-b) increase, augmenting the equations of motion as

$$
\begin{array}{r}
m_{p} \ddot{p}_{p}+m_{b} \ddot{p}_{f}-m_{p} g-m_{f} g+R_{\mathcal{P J}} m_{p} g=F_{s} \\
J_{p}{ }^{\mathcal{P}} \dot{\omega}_{p}+\omega_{p} \times\left(J_{p} \omega_{p}\right)-d_{p} \times\left(m_{p} \ddot{p}_{p}+m_{p} g\right) \ldots \\
+J_{f}{ }^{\mathcal{F}} \dot{\omega}_{f}+\omega_{f} \times\left(J_{f} \omega_{f}\right)-d_{f} \times\left(m_{f} \ddot{p}_{f}+m_{f} g\right)=T_{s}
\end{array}
$$

where

1. $J_{f} \in \mathbb{R}^{3} \times \mathbb{R}^{3}$ is the foot inertia tensor measured at its CG.

2. $\omega_{f} \in \mathbb{R}^{3}$ is the angular velocity of the $\mathcal{F}$ frame.

3. $d_{f} \in \mathbb{R}^{3}$ is the distance from the $\mathcal{F}$ origin to the foot CG in the $\mathcal{F}$ frame.

4. the left superscript $\mathcal{F}$ denotes a derivative on the non-inertial frame $\mathcal{F}$.

\subsection{Methodology}

\subsubsection{Experimental Setup}

The experiment setup was the same used in [14], except that 1) the motion capture sampling rate was set to $350 \mathrm{~Hz}, 2$ ) the data acquisition (DAQ) measuring the FP measurements was 
replaced by an NI USB-6251, National Instruments, USA; and 3) the DAQ and the motion captures were hardware-synchronized via the Optitrack eSync device.

FP Inertia Experiment. Both the linear actuators of the vibrating platform were actuated for 60 seconds with a uniformly distributed random signal, ranging the full actuation span. The update frequency of the actuators was set to 29,40 , and $67 \mathrm{~Hz}$ for a total of 3 runs.

Body Inertia Experiment. The same protocol for the FP Inertia Experiment was used. The mockup foot was represented by a calibrated weight (Error! Reference source not found..a) whose inertia parameters were derived from its CAD model: $22.68 \mathrm{~kg}$ mass, $(81.1,127.0,137.2)^{\mathrm{T}} 10^{-3} \mathrm{~kg} \mathrm{~m}^{2}$ moment of inertia, and $\left(\mathrm{J}_{\mathrm{yz}}, \mathrm{J}_{\mathrm{xz}}, \mathrm{J}_{\mathrm{xy}}\right)=(81.1,127.0,137.2)^{\mathrm{T}}$ $10^{-3} \mathrm{~kg} \mathrm{~m}^{2}$ product of inertia. The foot was fixed to the center of the FP by a mounting tape.

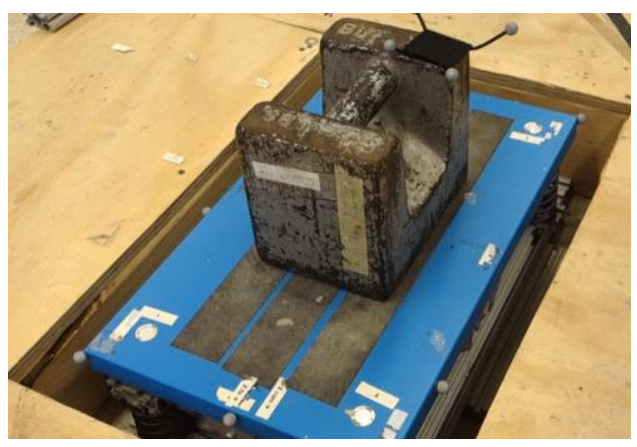

(a)

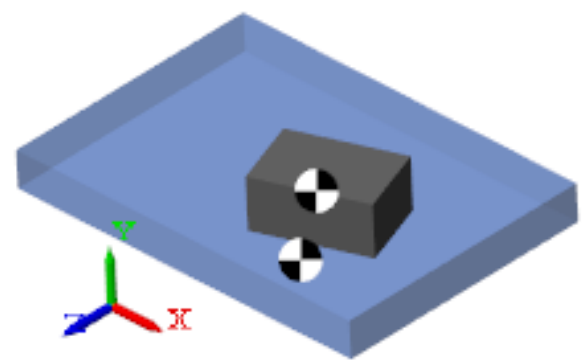

(b)

Figure 2. (a) Mockup foot represented by a calibrated weight. (b) Animation of the dynamic simulation. The axes convention matches the experimental setup.

\subsubsection{Dynamic Simulation}

A numerical simulation (Figure 2.b) was developed with Simscape Multibody software, MathWorks, USA, to validate the analytical description of the instrumented vibrating platform and to test whether the inertia parameters are theoretically observable. Since the source of error of parameter estimation can be either bad modeling or bad experimental measurements, this simulation is used to generate ideal measurements. Nevertheless, nonideal measurements and other limitations present in the real setup were also modeled to assess how they affect the performance of the estimation.

The simulation generates data in the same format as the experimental setup so that the same post-processing calculations are applied to both datasets. All measurements were added a normally distributed random noise of standard deviation of $0.1 \mathrm{~mm}, 10 \times 10^{-6} \mathrm{rad}, 0.1 \mathrm{~N}$, $0.05 \mathrm{Nm}$, for all translations, Euler angle rotations (YZX), forces, and torques, respectively. To assess the sensibility of the inertia estimation method due to noise, the system was simulated 20 times to calculate the average and standard deviation of the parameter estimates. 
Other sources of error were added to the simulation to assess how they affect the performance of the parameter estimation. The simulated FP force measurement was subtracted a tare weight on the normal axis and an unobservable inertia was added under the FP representing the upper frame of the vibrating platform $\left(9 \mathrm{~kg}\right.$ mass, $(10,50,5)^{\mathrm{T}} \mathrm{mm}$ CG from the universal joint, $(0.9,0.9,0.3)^{\mathrm{T}} \mathrm{kg} \mathrm{m}^{2}$ moment of inertia, $(0.2,0.1,0.1)^{\mathrm{T}} \mathrm{kg} \mathrm{m}^{2}$ product of inertia). Rotational springs ( $270 \mathrm{Nm} / \mathrm{deg}$ in the $Z$ axis, $150 \mathrm{Nm} / \mathrm{deg}$ in $\mathrm{X}$ axis) were added to the universal joint [14].

The analytical model of the system, equations of motion (1.a-b) and (2.a-b), was compared to the numerical model. The output kinematic data of the numerical model was inserted on the analytical model and the output torques have shown to be equal. Therefore, validating the analytical description of the vibrating platform.

\subsubsection{Parameter Estimation Algorithm}

Signal Processing. Both the kinematics and dynamics measurements were low-pass filtered $\left(40 \mathrm{~Hz}\right.$ cutoff, $100^{\text {th }}$ order, Hanning window-based). The derivatives were numerically calculated via a Savitzky-Golay filter [20] $\left(12^{\text {th }}\right.$ order polynomial, 13-point window) to account for high-frequency signal components.

Residual of FP Inertia Estimation. To estimate $J_{p}$ and $d_{p}$, a residual variable is defined subtracting the right-hand side from the left-hand side of equations of motion (1.a) and (1.b), obtaining

$$
\varepsilon\left(J_{p}, d_{p}\right)=\left(\begin{array}{c}
m_{p} \tilde{\ddot{p}}_{p}-m_{p} g+\tilde{R}_{P I} m_{p} g-\widetilde{F}_{s} \\
{ }_{p}{ }^{P} \widetilde{\dot{\omega}}_{p}+\widetilde{\omega}_{p} \times\left(J_{p} \widetilde{\omega}_{p}\right)-d_{p} \times\left(m_{p} \tilde{\ddot{p}}_{p}+m_{p} g\right)-\widetilde{T}_{s}
\end{array}\right)
$$

where the tilde accent refers to the uncertain measurements. The mass is not estimated because it can be measured directly by the FP after it is tared with the FP on the vertical orientation. That is, while the weight is not acting in the normal direction of the FP.

Residual of Body Inertia Estimation. Similarly, to estimate $m_{b}, J_{b}$, and $d_{p}$, another residual variable is defined subtracting the right-hand side from the left-hand side of equations of motion (2.a) and (2.b), obtaining

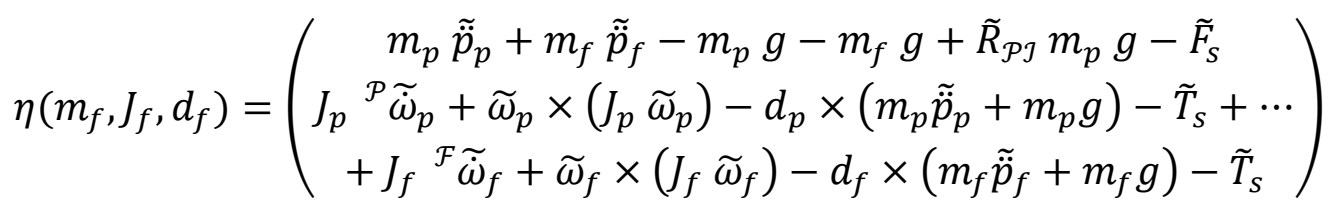

Different from $\varepsilon$, the mass property was included as an unknown variable on the residual $\eta$ to verify that the system can estimate all 10 inertia components simultaneously. That will be necessary when estimating the real foot mass, which cannot be directly measured. 
Nonlinear Optimization. The unknown parameters were estimated such that the residuals are minimized according to a cost function. The cost function takes into consideration all the $N$ samples of the dataset and the uncertainty of each residual vector element to form

$$
\begin{gathered}
\left(J_{p}, d_{p}\right)=\min _{\left(J_{p}, d_{p}\right)} \frac{1}{N} \sum_{k=1}^{N} \lambda^{T}\left|\varepsilon_{k}\left(J_{p}, d_{p}\right)\right| \\
\left(m_{f}, J_{f}, d_{f}\right)=\min _{\left(m_{f}, J_{f}, d_{f}\right)} \frac{1}{N} \sum_{k=1}^{N} \lambda^{T}\left|\eta_{k}\left(m_{f}, J_{f}, d_{f}\right)\right|
\end{gathered}
$$

where $\varepsilon_{k} \in \mathbb{R}^{6}$ and $\eta_{k} \in \mathbb{R}^{6}$ are residuals at sample $k$, and $\lambda=(1,1,1,2,2,2)^{T}$ is a weight vector. This weight vector should be inversely proportional to the uncertainty of the equations of motion, considering the noisy measurements. Finally, the unknown parameters are estimated minimizing the cost functions (5) and (6) via a trust region method based on interior point [21]. The FP inertia parameters are estimated first, so that $J_{p}$ and $d_{p}$ estimates are fed into equation (4), to compute the $m_{f}, J_{f}$, and $d_{f}$ parameters.

\subsection{Results and Discussion}

FP Inertia Estimates. The FP inertia estimates showed a small deviation between experiments (Table 1) and they agree with a homogeneous cuboid with the same mass and dimensions. However, the inertia parameters should not necessarily match the homogeneous cuboid because the FP might have heterogeneous mass distribution. Altogether, the estimates indicate the algorithm has high accuracy and consistency.

\begin{tabular}{|c|c|c|c|c|c|c|c|}
\hline \multirow{3}{*}{ Parameter } & \multirow{3}{*}{$\begin{array}{l}\text { Ideal } \\
\text { Cuboid }\end{array}$} & \multirow{2}{*}{\multicolumn{3}{|c|}{$\begin{array}{c}\text { Experimental } \\
\text { Input Bandwidth }\end{array}$}} & \multicolumn{3}{|c|}{ Simulation } \\
\hline & & & & & \multirow{2}{*}{ Reference } & \multicolumn{2}{|c|}{ Absolute Error } \\
\hline & & 29 & 40 & 67 & & w/ noise & no noise \\
\hline $\mathrm{kg}$ & 4.64 & 4.64 & 4.64 & 4.64 & 4.64 & $0.00 \pm 0.00$ & 0.00 \\
\hline $\mathrm{mm}$ & 0.0 & 5.5 & 7.2 & 2.4 & 5.03 & $0.06 \pm 0.01$ & -0.00 \\
\hline $\mathrm{mm}$ & -14.4 & -29.9 & -27.3 & -21.4 & -26.20 & $-0.31 \pm 0.20$ & 0.00 \\
\hline $\mathbf{z}_{\mathbf{0}} \quad \mathrm{mm}$ & $\mathbf{0}$ & -0.9 & -0.9 & -1.1 & -0.97 & $0.03 \pm 0.01$ & 0.00 \\
\hline $\boldsymbol{J}_{\boldsymbol{x}} \quad g m^{2}$ & 34.8 & 41.0 & 40.4 & 39.8 & 440.40 & $-0.43 \pm 0.07$ & -0.00 \\
\hline$J_{y} \quad g m^{2}$ & 131.1 & 113.4 & 102.6 & 138.2 & 118.07 & $-0.60 \pm 6.72$ & 0.01 \\
\hline $\boldsymbol{J}_{\mathbf{z}} \quad g \mathrm{~m}^{2}$ & 97.0 & 105.5 & 106.7 & 103.6 & 105.27 & $1.03 \pm 0.23$ & 0.00 \\
\hline $\boldsymbol{J}_{\boldsymbol{y z}} \quad g m^{2}$ & 0.0 & -0.6 & 2.5 & 2.2 & 1.37 & $0.04 \pm 0.01$ & 0.00 \\
\hline $\boldsymbol{J}_{\boldsymbol{x} \boldsymbol{z}} \quad g m^{2}$ & 0.0 & -2.4 & 2.1 & -1.1 & -0.47 & $-0.23 \pm 0.06$ & 0.00 \\
\hline $\boldsymbol{J}_{x y} \quad g m^{2}$ & 0.0 & 13.0 & 9.1 & 2.3 & 8.13 & $-0.08 \pm 0.03$ & 0.00 \\
\hline
\end{tabular}

Table 1. Inertial parameters estimates of the FP. 
The system was simulated using the average of the experimental inertial estimates. When the measurements were not added noise and not filtered (Table 1, no noise), the estimation errors were virtually zero. Otherwise, when the measurements were added noise (Table 1, $\mathrm{w} /$ noise), all the parameters were estimated with fair accuracy and the moment of inertia on the Y-axis had large deviation, but average close to the reference value. To assess the sensibility of the estimation given the sensor noise,

Foot Inertia Estimates. Using the experimental data, the estimation was fairly accurate for all parameters, except $J_{y}$ and $J_{x}$ (Table 2). The source of the $J_{y}$ error is possibly because the platform does not rotate on the $\mathrm{Y}$ axis much, thus, not exciting the foot inertia on the $\mathrm{Y}$ axis. Further analysis should be done to explain the source of the $J_{x}$ error.

Table 2. Inertial parameters estimates of the mockup foot.

\begin{tabular}{cc|c|ccc||cc}
\hline \multirow{2}{*}{ Parameter } & \multirow{2}{*}{ Reference } & \multicolumn{3}{|c|}{ Experimental } & \multicolumn{2}{c}{ Simulation } \\
\cline { 3 - 8 } & & $\mathbf{2 9}$ & $\mathbf{4 0}$ & $\mathbf{6 7}$ & \multicolumn{2}{c}{$\begin{array}{c}\text { Absolute Error } \\
\text { whoise }\end{array}$} & no noise \\
\hline $\boldsymbol{m}$ & $\mathrm{kg}$ & $\mathbf{2 2 . 6 8}$ & 22.98 & 23.04 & 23.10 & $-3.58 \pm 0.5$ & -0.00 \\
\hline $\boldsymbol{x}_{\mathbf{0}}$ & $m m$ & $\mathbf{0 . 0}$ & -0.3 & -0.3 & -0.1 & $-0.0 \pm 0.0$ & -0.0 \\
\hline $\boldsymbol{y}_{\mathbf{0}}$ & $m m$ & $\mathbf{- 9 0 . 7}$ & -73.1 & -72.5 & -73.9 & $2.5 \pm 0.1$ & 0.2 \\
\hline $\boldsymbol{z}_{\mathbf{0}}$ & $m m$ & $\mathbf{0 . 0}$ & -0.73 & -0.9 & -1.0 & $-0.0 \pm 0.0$ & 0.0 \\
\hline $\boldsymbol{J}_{\boldsymbol{x}}$ & $g m^{2}$ & $\mathbf{8 1 . 1}$ & 0.0 & 0.0 & 0.0 & $-11.7 \pm 0.4$ & -0.5 \\
\hline $\boldsymbol{J}_{\boldsymbol{y}}$ & $g m^{2}$ & $\mathbf{1 2 7 . 0}$ & 13.6 & 169.8 & 200.5 & $121.7 \pm 57.2$ & -133.9 \\
\hline $\boldsymbol{J}_{\boldsymbol{z}}$ & $g m^{2}$ & $\mathbf{1 3 7 . 2}$ & 146.4 & 145.8 & 159.7 & $-6.4 \pm 0.6$ & -0.9 \\
\hline $\boldsymbol{J}_{\boldsymbol{y} \boldsymbol{z}}$ & $g m^{2}$ & $\mathbf{0 . 0}$ & -1.1 & -1.5 & -0.6 & $-0.2 \pm 1.1$ & -0.2 \\
\hline $\boldsymbol{J}_{\boldsymbol{x} \boldsymbol{z}}$ & $g m^{2}$ & $\mathbf{0 . 0}$ & 2.0 & -1.4 & -0.0 & $-0.6 \pm 0.3$ & -0.1 \\
\hline $\boldsymbol{J}_{\boldsymbol{x y}}$ & $g m^{2}$ & $\mathbf{0 . 0}$ & 4.4 & 2.9 & 2.8 & $0.0 \pm 1.1$ & 0.6 \\
\hline
\end{tabular}

On the other hand, the results from the simulation showed small errors, except for $J_{y}$. This large and small estimation errors on the experimental and simulated analysis, respectively, suggests the source of error is in the sensor measurements, on the physical model, or noise model.

Further work is necessary to improve the inertia estimation accuracy of the FP and the foot, and to extend the method to the full ankle impedance estimation. The inaccurate experimental estimation of the foot inertia also suggests both the FP inertia and mockup foot experiments might be revised. 


\section{Wearable Vision System for Ground Mapping}

Conventional sensors have been used to classify the walking modes executed by prosthetics users, such as level walk, standing, stair ascent and descent, and ramp ascent and descent. $\mathrm{Li}$ and Hsiao-Wecksler [22] classified the walking mode from pose thresholds of a foot orthosis, but their approach could not predict future footsteps in time. Other machine learning algorithms were used to predict the walking mode, using a dynamic Bayesian Network [3] and Gaussian Mixture Models [2], but both have shown limitations identifying correctly and timely the transitions between walking modes.

The accuracy of the walking mode transitions can be improved introducing knowledge of the environment by a vision system. Du et al. [23] showed that a correct prior knowledge of the terrain in front of the user not only decrease their classification error but also allowed earlier prediction. Many techniques have been used to identify the terrain in front of the user, such as using a laser sensor [24], or a Microsoft Kinect sensor [8]. These results show great promise of the vision systems to increase the mobility of prosthesis and orthosis users.

This chapter presents an overview of depth sensing devices, listing some that can be used to assist prosthesis control. It also describes a prototype called GaitEyes, a wearable device attached to the prosthesis user's hip that map the ground and localize it on an inertial frame. The description of its components and the algorithms for localization and plane fitting are described next.

\subsection{Background}

The recent technological maturation of depth sensing devices, the advancement in computing power, and algorithms efficiency has empowered machines with depth perception. This section describes technologies of depth sensing devices proper for wearable device application and later presents the state-of-the-art algorithms for depth perception.

\subsubsection{Depth Sensing Devices}

Structured Light. Structured light devices contain a camera and a light pattern projector, which in most cases work on the infrared spectrum. This type of device senses depth based on the warping of the projected pattern onto the environment. Devices that could be used as a wearable ground mapping system are the Occipital Structure, Intel RealSense R200 and SR300.

Because it depends on infra-red light, structured light cameras are sensitive to interference from sunlight, limiting outdoor applications. On the other hand, they show good performance on any type of surface, including homogeneous ones, like a blank floor. Finally, it is computationally efficient because it works on binary rather than intensity or color images. 
Stereo Vision. Stereoscopy uses the instantaneous views from two cameras to triangulate the position of a point. A common setup uses two cameras of the same model mounted on a rigid base, in parallel, and facing the same direction. For additional depth accuracy, these two cameras might include a circuit to synchronize their image frames (frames of each camera are created simultaneously) and global shutters (all pixels within a frame are created simultaneously). Devices that could be used as a wearable ground mapping system are the Minoru 3D Webcam, StereoLabs ZED, and the Leap Motion.

One benefit of the stereo cameras is that it works well in environments the human eye can see, including sunlit environments, because it depends on the light source from the environment. Also, because stereo systems use popular cameras in an advanced technological state, the components are low-cost and lightweight. The downside of stereo cameras is that it can only perceive a semi-dense depth map because it fails on homogeneous surfaces. Furthermore, it requires high computation effort because stereo algorithms process intensity images and rely on image search.

Time of Flight. Time of flight (TOF) devices emit an unstructured light source onto a surface and measures the travel time of the light ray. The depth is computed from the known speed of light and time delay from emission to detection. Devices that could be used as a wearable ground mapping system are the SoftKinetic DepthSense 525 and the CamBoard Pico Flexx.

TOF devices are further classified by its light sensitive sensor. Light Detection and Ranging (LiDAR) are TOF devices with a single light sensitive sensor but can scan a line or area by reflecting the emitted and received light by a rotating mirror. Using matrix shaped sensors like in regular cameras, Flash LiDAR devices achieve higher scanning rates.

In conclusion, the TOF hardware is superior to the other depth sensing technologies except for its low performance in outdoor environments and overall depth accuracy. But newer generations of sensors are expected to solve these challenges [25]. Also, because wearables must be carried around and are susceptible to impacts, it should be lightweight and easily replaceable, making stereo system also a good option for this application.

\subsubsection{Simultaneous Localization and Mapping}

Simultaneous Localization and Mapping (SLAM) solves a common problem in mobile robotics, of navigating in an unknown environment, which requires the construction of the environment map and use this model to localize itself as the robot moves [26]. SLAM minimizes map inconsistencies correcting measurements from ranging sensors and the robot pose.

The SLAM algorithms are further divided into Visual SLAM [27, 28] when using only exteroceptive sensors (measures external data, like depth sensors), or Visual-Inertial 
SLAM [29-32] when using both exteroceptive and proprioceptive sensors (measures robot's kinetic states, like heading, velocity, acceleration).

Furthermore, the depth and inertial sensor might be tightly or loosely coupled, depending if their error minimizations are performed jointly or sequentially, respectively. Since the first approach has the flexibility to balance inertial sensor biases and depth noise, it has shown to be more accurate [29]. But due to the higher dimensionality, the computation cost is higher [32].

Finally, SLAM can be classified as direct or indirect depending if images are reduced to a set of key-points. Direct SLAM compares images, either over time from the same camera or over different camera frames, using the intensity images directly. The use of the entire image for mapping estimates a more dense depth map, comparing to indirect approaches [31]. On the other hand, indirect methods identify and convert salient points to be tracked over time.

Image processing has the problem of large dimensionality, so image tracking solutions generally use local salient features as a reduced subset of information. These features are parts of the image that differs from its neighborhood, such as corners, edges, and region blobs. The features are represented in another space using Feature Descriptors for ease of comparison to one another and allow insensibility against change in scale, rotation, warp, and translation [33].

SLAM can be a valuable tool for prosthesis control perception, both for the reconstructed map and the pose estimates. The reconstructed map is more accurate than the depth map generated by the depth sensing devices alone, which enables a better ground reconstruction. Also, the pose estimate improves the conversion of the camera measurements to the inertial frame, also reducing reconstruction errors.

\subsection{Ground Fitting Algorithm}

The GaitEyes device (Figure 3.a) is composed of a stereo camera (StereoLabs ZED), an IMU (InvenSense MPU-6050), and a mobile computer (NVIDIA Jetson TX1). The mobile computer samples the camera measurements at $20 \mathrm{fps}$ (720p resolution) and the IMU at $100 \mathrm{~Hz}$.

The camera is attached to the user's hip and pitched 45 degrees down to record the foothold region of the next step (Figure 3.b). To account for the hip motion as the user moves, the IMU, rigidly connected to the center of the stereo camera, records the camera orientation. The position and orientation of the IMU in respect to the camera are obtained through an extrinsic calibration [34]. 


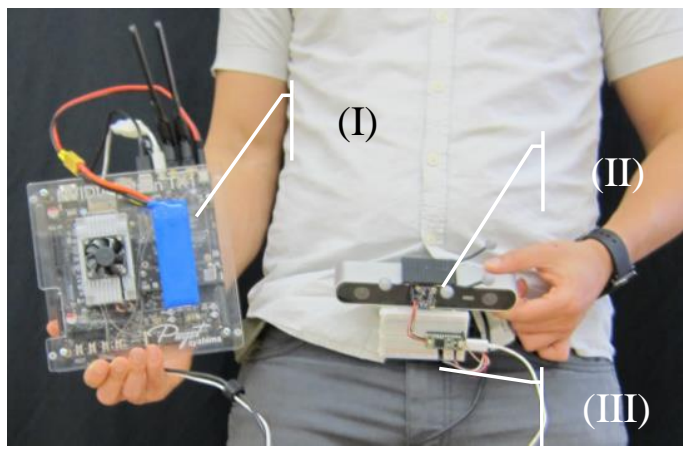

(a)

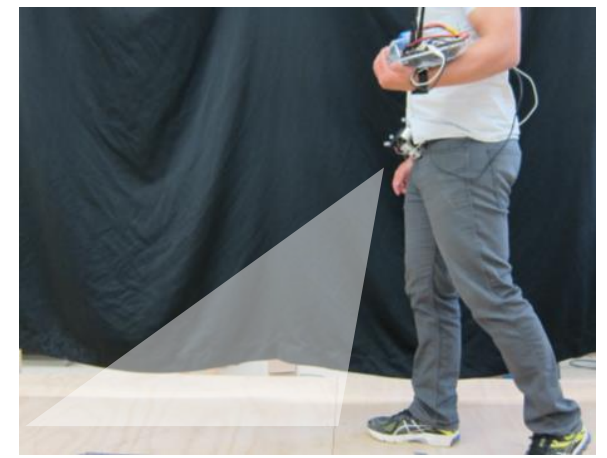

(b)

Figure 3. (a) GaitEyes components: stereo camera (I), IMU (II), and mobile computer (III). (b) GaitEyes on subject, pointing to the ground.

To estimate the user's pose and the ground slope and height, the mobile computer 1) estimates the pose of the camera, 2) creates a depth map of the environment, 3) identifies a plane model for the foothold region, and 4) update the plane model estimate over time. All these steps are repeated continuously $(20 \mathrm{~Hz})$ so that past iterations can improve the accuracy of the foothold region.

The pose estimation of the camera uses a Simultaneous Localization and Mapping (SLAM) algorithm provided with the ZED camera. This SLAM algorithm balances the uncertainty of the image captures to estimate the most likely camera displacement while keeping the depth map consistent over continuous samples. Although the SLAM algorithm also results in a consistent depth map of the environment, the map cannot be directly used for the ground reconstruction because it is not dense.

Instead, the depth map of the environment is computed with a semi-global matching stereo algorithm [30]. Like other stereo vision algorithms, it uses synchronized captures of two cameras to triangulate the position of each point in the field of view. The output of the stereo matching is an image, with each pixel representing the distance from the camera to the captured surface (Figure 4.a).

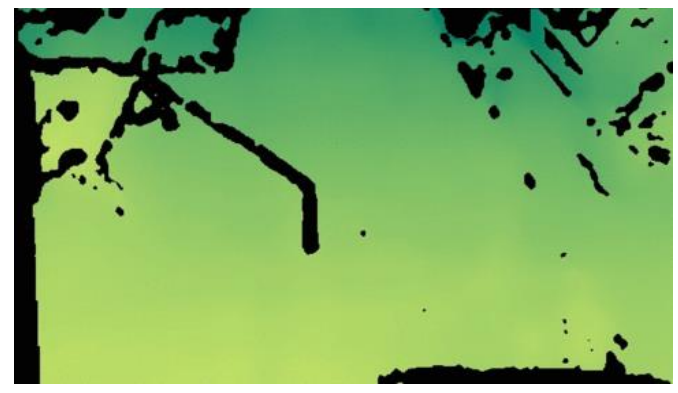

(a)

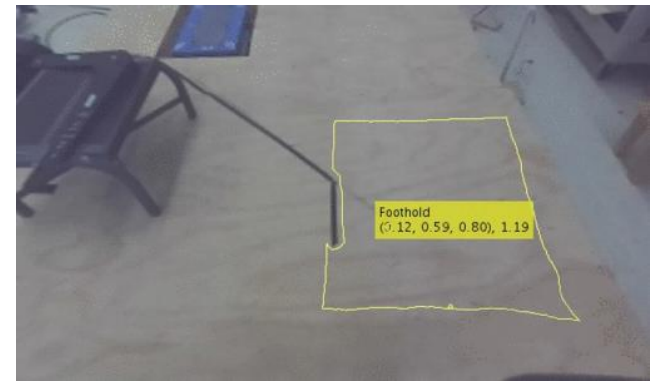

(b)

Figure 4. (a) Depth map from the image capture. (b) Selected region of interest for the ground plane estimation. 
The set of points representing the foothold region of the next step (Figure 4.b) is modeled as a plane (Eq. 7), parameterized by a normal vector and z-intercept. These parameters are estimated using the Least Square Method (Eq. 8) according to the plane equation.

$$
\begin{gathered}
{\left[\left(\begin{array}{l}
x \\
y \\
z
\end{array}\right)-\left(\begin{array}{c}
0 \\
0 \\
z_{0}
\end{array}\right)\right]^{T}\left(\begin{array}{l}
a \\
b \\
1
\end{array}\right)=0} \\
\left(\begin{array}{ccc}
x_{1} & y_{1} & -1 \\
x_{2} & y_{2} & -1 \\
\vdots & \vdots & \vdots \\
x_{N} & y_{N} & -1
\end{array}\right)\left(\begin{array}{c}
a \\
b \\
z_{0}
\end{array}\right)=\left(\begin{array}{c}
-z_{1} \\
-z_{2} \\
\vdots \\
-z_{N}
\end{array}\right)
\end{gathered}
$$

where

1. $(x, y, z)^{T} \in \mathbb{R}^{3}$ is the point on the plane.

2. $(a, b, 1)^{T} \in \mathbb{R}^{3}$ is a normal vector to the plane.

3. $z_{0} \in \mathbb{R}$ is the z-intercept of the plane.

To convert these parameters from the camera to the inertial frame, the camera pose estimated by the SLAM or by the IMU is used. This reference conversion allows the update of the ground estimate as new data arrives, considering the motion of the camera.

\subsection{Discussion}

Qualitative tests indicate GaitEyes can estimate the ground slope in an indoor environment. The camera and the IMU were interfaced and each intrinsically calibrated. The system estimated the normal vector and height of the ground while a healthy person walked in a flat walkway.

The performance of ground identification must be evaluated quantitatively. A first experiment should identify different ground slopes while the camera is static or moving very slowly. This initial test will verify 1) biases on the IMU orientation, 2) depth accuracy of the range imager, and 3) the extrinsic calibration between camera and IMU, that is, the frame conversion from camera to inertial frame. A second test will verify if the system is accurate for fast camera motions common during walk. This test might reveal 1) degraded image quality due to motion blur, 2) effect of fast motion on the IMU orientation estimates, and 3) synchronization errors between IMU and camera. 


\section{Future Work}

At the completion of the Ph.D. work, GaitEyes must be able to estimate ground properties, such as slope and step height, and the ankle impedance must be known for each of these properties. The estimation accuracy and processing time should be sufficient for implementation in a powered ankle-foot prosthesis.

For the environment perception, other depth sensing and localization solutions should be explored. The stock SLAM algorithm provided by the ZED camera might be replaced by a visual-inertial alternative. To decrease the size of the system, the range imager might be replaced by a TOF camera.

For the ankle impedance estimation, the analytical model and the simulation will be added a stiffness and damping torques to represent the ankle impedance. The simulation will validate the equations of motion, provide a reference value when estimating parameters, and indicate how observable the parameters are with the presence of noise. Parallelly, human trials will be recorded for a set of gait maneuvers and ground slopes. 


\section{Conclusion}

Intelligent active prosthesis makes use of environment and user intention perception to control the actuators. Although much work have been directed to understand the user intention, the estimation of the environment for prosthesis control is rather limited. And worse yet, the knowledge of the environment will not play a significant role to prosthesis performance until there are studies relating the impedance of the ankle to the environment properties. This report describes a preliminary work addressing these issues with 1) a vision system that estimates the ground slope and 2) a system identification algorithm for the vibrating platform to estimate the time-varying ankle impedance during gait maneuvers.

To develop the vision system, a detailed research on range sensing technologies and map reconstruction algorithms was made. The main technologies are the structured light, stereo vision, and time of flight. The stereo vision was selected for this project because it works well on outside environment, is low-cost, and easily replaceable. The vision system could estimate the ground as a plane model and convert the normal vector of the plane to the inertial frame using IMU orientation measurements.

The impedance estimation algorithm was first validated as a simple inertia identification problem and limitations of the FP hardware was discovered in initial tests. The inertial properties of the FP were accurately estimated using simulation data (moments of inertia on the vertical axis were not observable), and different experimental runs resulted in consistent estimations. But accurate inertial estimation of the a mockup foot was only possible in the simulation and for a few parameters, for the experimental case. Further work will revise the experimental protocol to reduce the estimation errors.

The future steps of this project will quantitively evaluate the performance of the vision system for different ground settings. And, for each of these grounds settings, the ankle impedance will be evaluated with the vibrating platform. 


\section{References}

1. Tucker, M.R., et al., Control strategies for active lower extremity prosthetics and orthotics: a review. Journal of neuroengineering and rehabilitation, 2015. 12: p. 1.

2. Varol, H.A., F. Sup, and M. Goldfarb, Multiclass real-time intent recognition of a powered lower limb prosthesis. IEEE Transactions on Biomedical Engineering, 2010. 57(3): p. 542-551.

3. Young, A.J., et al., Intent Recognition in a Powered Lower Limb Prosthesis Using Time History Information. Annals of Biomedical Engineering, 2014. 42(3): p. 631641.

4. Shultz, A.H., B.E. Lawson, and M. Goldfarb, Variable Cadence Walking and Ground Adaptive Standing With a Powered Ankle Prosthesis. Ieee Transactions on Neural Systems and Rehabilitation Engineering, 2016. 24(4): p. 495-505.

5. McIntosh, A.S., et al., Gait dynamics on an inclined walkway. Journal of biomechanics, 2006. 39(13): p. 2491-2502.

6. Voloshina, A.S., et al., Biomechanics and energetics of walking on uneven terrain. Journal of Experimental Biology, 2013. 216(21): p. 3963-3970.

7. Sup, F., H.A. Varol, and M. Goldfarb, Upslope walking with a powered knee and ankle prosthesis: initial results with an amputee subject. IEEE Transactions on Neural Systems and Rehabilitation Engineering, 2011. 19(1): p. 71-78.

8. Krausz, N.E., T. Lenzi, and L.J. Hargrove, Depth Sensing for Improved Control of Lower Limb Prostheses. Ieee Transactions on Biomedical Engineering, 2015. 62(11): p. 2576-2587.

9. Liu, M., D. Wang, and H. Huang, Development of an Environment-Aware Locomotion Mode Recognition System for Powered Lower Limb Prostheses. Ieee Transactions on Neural Systems and Rehabilitation Engineering, 2016. 24(4): p. 434-443.

10. Au, S.K., et al. Powered Ankle-Foot Prosthesis for the Improvement of Amputee Ambulation. in International Conference of the IEEE, Engineering in Medicine and Biology Society. 2007. Lyon.

11. Ficanha, E.M., et al., Time-Varying Human Ankle Impedance in the Sagittal and Frontal Planes during Stance Phase of Walking, in IEEE International Conference on Robotics and Automation (ICRA). 2017: Singapore. 
12. Lee, H. and N. Hogan, Time-Varying Ankle Mechanical Impedance During Human Locomotion. IEEE Transactions on Neural Systems and Rehabilitation Engineering, 2015. 23(5): p. 755-764.

13. Rouse, E., et al., Estimation of Human Ankle Impedance During Walking Using the Perturberator Robot, in Fourth IEEE RAS/EMBS International Conference on Biomedical Robotics and Biomechatronics. 2012: Roma, Italy.

14. Ficanha, E.M., G.A. Ribeiro, and M. Rastgaar, Design and Evaluation of a 2-DOF Instrumented Platform for Estimation of the Ankle Mechanical Impedance in the Sagittal and Frontal Planes. IEEE/ASME Transactions on Mechatronics, 2016. 21(5): p. 2531-2542.

15. Vlutters, M., et al., Direct measurement of the intrinsic ankle stiffness during standing. Journal of biomechanics, 2015. 48(7): p. 1258-1263.

16. Loram, I.D. and M. Lakie, Direct measurement of human ankle stiffness during quiet standing: the intrinsic mechanical stiffness is insufficient for stability. Journal of Physiology, 2002. 545(3): p. 1041-1053.

17. Schedlinski, C. and M. Link, A survey of current inertia parameter identification methods. Mechanical systems and signal processing, 2001. 15: p. 189-211.

18. Füllekrug, U. and C. Schedlinski. Inertia Parameter Identification from Base Excitation Test Dat. in Environmental Testing for Space Programmes. 2004.

19. Fregolent, A. and A. Sestieri, Identification of rigid body inertia properties from experimental data. Mechanical systems and signal processing, 1996. 10(6): p. 697709.

20. Savitzky, A. and M.J. Golay, Smoothing and differentiation of data by simplified least squares procedures. Analytical chemistry, 1964. 36(8): p. 1627-1639.

21. Byrd, R.H., J.C. Gilbert, and J. Nocedal, A trust region method based on interior point techniques for nonlinear programming. Mathematical Programming, 2000. 89(1): p. 149-185.

22. Li, Y.D. and E.T. Hsiao-Wecksler. Gait mode recognition and control for a portable-powered ankle-foot orthosis. 2013. IEEE.

23. Du, L., et al., Toward design of an environment-aware adaptive locomotion-moderecognition system. IEEE Transactions on Biomedical Engineering, 2012. 59: p. 2716-2725.

24. Zhang, F., et al. Preliminary design of a terrain recognition system. 2011. IEEE. 
25. Bartels, J., 2D or 3D Camera? Which 3D Camera Technology Fits Your Application?, B. AG, Editor.: Germany.

26. Dissanayake, M.G., et al., A solution to the simultaneous localization and map building (SLAM) problem. IEEE Transactions on robotics and automation, 2001. 17(3): p. 229-241.

27. Engel, J., T. Schöps, and D. Cremers. LSD-SLAM: Large-scale direct monocular SLAM. in European Conference on Computer Vision. 2014. Springer.

28. Mur-Artal, R. and J.D. Tardos, ORB-SLAM2: an Open-Source SLAM System for Monocular, Stereo and RGB-D Cameras. arXiv preprint arXiv:1610.06475, 2016.

29. Leutenegger, S., et al., Keyframe-based visual-inertial odometry using nonlinear optimization. International Journal of Robotics Research, 2015. 34(3): p. 314-334.

30. Mur-Artal, R. and J.D. Tardos, Visual-inertial monocular SLAM with map reuse. arXiv preprint arXiv:1610.05949, 2016.

31. Usenko, V., et al. Direct Visual-Inertial Odometry with Stereo Cameras. in Int. Conf. on Robotics and Automation. 2016.

32. Weiss, S. and R. Siegwart. Real-time metric state estimation for modular visioninertial systems. in Robotics and Automation (ICRA), 2011 IEEE International Conference on. 2011. IEEE.

33. Li, L., Time-of-flight camera-an introduction.

34. Furgale, P., J. Rehder, and R. Siegwart. Unified temporal and spatial calibration for multi-sensor systems. in 2013 IEEE/RSJ International Conference on Intelligent Robots and Systems. 2013. 